\title{
BIBLIOGRAPHY
}

1. Erwin Kleinfeld, Right alternative rings, Proc. Amer. Math. Soc. vol. 4 (1953) pp. 939-944.

2. R. L. San Soucie, Right alternative division rings of characteristic two, Proc. Amer. Math. Soc. vol. 6 (1955) pp. 291-296.

3. L. A. Skornyakov, Right alternative fields, Izvestia Akad. Nauk SSSR Ser. Mat. vol. 15 (1951) pp. 177-184.

The University of Oregon

\section{ON AN ITERATIVE PROCEDURE FOR OBTAINING THE PERRON ROOT OF A POSITIVE MATRIX}

\section{RICHARD BELLMAN}

1. Introduction. The purpose of this paper is to present a new iterative procedure for obtaining the characteristic root of largest absolute value of a positive matrix.

The origin of the method is as follows. There is a result of von Neumann [7], a generalization of his fundamental min-max theorem in the theory of games [8], to the effect that

$$
\operatorname{Min}_{y} \operatorname{Max}_{x} \frac{(x, A y)}{(x, B y)}=\underset{x}{\operatorname{Max}} \underset{y}{\operatorname{Min}} \frac{(x, A y)}{(x, B y)}
$$

where the variation is over the region defined by

$$
R \text { : }
$$

$$
\text { (a) } x_{i} \geqq 0, \quad \sum_{i=1}^{n} x_{i}=1,
$$

$$
\text { (b) } y_{i} \geqq 0, \quad \sum_{i=1}^{n} y_{i}=1 \text {, }
$$

and it is assumed that $B$ has the property that

$$
(x, B y) \geqq b>0
$$

for all $(x, y) \in R$.

It was observed by Shapley [6] that this result can be obtained as a by-product of the theory of "games of survival," cf. $[1 ; \mathbf{5} ; \mathbf{6}]$, which requires only the fundamental min-max theorem, by considering the equation for $\lambda$,

Received by the editors November 22, 1954. 


$$
\begin{aligned}
\lambda & =\operatorname{Min}_{y} \operatorname{Max}_{x}[(x, A y)+\lambda(1-(x, B y))] \\
& =\underset{x}{\operatorname{Max}} \operatorname{Min}_{y}[(x, A y)+\lambda(1-(x, B y))],
\end{aligned}
$$

where we impose the additional assumption the $1>(x, B y)$ for all $(x, y) \in R$. This restriction is of no importance because of the homogeneity of the ratio in (1).

It is then easy to prove that there is a unique solution of (3) which may be obtained as the limit of the sequence $\left\{\lambda_{n}\right\}$ defined by

$$
\begin{aligned}
\lambda_{0} & =\underset{v}{\operatorname{Min}} \operatorname{Max}_{x}[(x, A y)]=\underset{x}{\operatorname{Max}} \underset{\nu}{\operatorname{Min}}[(x, A y)], \\
\lambda_{n+1} & =\underset{v}{\operatorname{Min}} \operatorname{Max}_{x}\left[(x, A y)+\lambda_{n}(1-(x, B y))\right] \\
& =\operatorname{Max}_{x} \operatorname{Min}_{\nu}\left[(x, A y)+\lambda_{n}(1-(x, B y))\right],
\end{aligned}
$$

and that this solution is then given also by the common value of the ratio in (1).

This procedure yields a theoretical and computational hold on $\lambda$, which is quite useful. Furthermore, by means of this ingenious device we have a means of linearizing a number of problems relating to ratios. In this paper we shall apply this idea to the problem of determining the root of a positive matrix of largest absolute value, using a variational representation for this root involving a ratio.

2. The Perron root. Let $A$ be a square matrix $\left(a_{i j}\right)$. It is called positive $e^{1}$ if $a_{i j}>0$ for all $i$ and $j$. The basic result concerning positive matrices is due to Perron and is the following:

Lemma 1. If $A$ is a positive matrix, there is a unique characteristic root of largest absolute value. This root is positive and its associated characteristic vector may be taken to be positive.

Notation. We denote this root by $p(A)$, the Perron root of $A$.

An alternative definition of this root, possessing the great merit of involving a variation, is

LEMMA 2.

$$
\begin{aligned}
p(A) & =\underset{x}{\operatorname{Max}} \operatorname{Min}_{\imath} \sum_{j=1}^{n} a_{i j} x_{j} / x_{i} \\
& =\operatorname{Min}_{x} \operatorname{Max}_{\imath} \sum_{j=1}^{n} a_{i j} x_{j} / x_{i} .
\end{aligned}
$$

${ }^{1}$ Not to be confused with positive definite. 
This result has been used by several authors independently, and does not seem to have any particular known origin. It was communicated to the author several years ago by $\mathrm{H}$. Bohnenblust in connection with a different problem, see [2].

Here the variation is over the region defined by

$$
x_{i} \geqq 0, \quad \sum_{i} x_{i}=1 \text {. }
$$

3. A refinement of Lemma 2. Let us show that Lemma 2 may be replaced by the stronger result

LEMMA 3. We have

$$
\begin{aligned}
p(A) & =\underset{R^{\prime}}{\operatorname{Max}} \underset{i}{\operatorname{Min}} \sum_{j=1}^{n} a_{i j} x_{j} / x_{i} \\
& =\underset{R^{\prime}}{\operatorname{Min}} \operatorname{Max}_{i} \sum_{j=1}^{n} a_{i j} x_{j} / x_{i},
\end{aligned}
$$

where $R^{\prime}$ is defined by

$$
x_{i} \geqq d, \quad \sum_{i} x_{i}=1,
$$

and $d$ is some parameter depending only upon A. Specifically, we may take

$$
d=\operatorname{Min}_{i, j} a_{i j} / \operatorname{Max}_{i}\left(\sum_{j=1}^{n} a_{i j}\right) .
$$

Proof. The minimizing $x_{i}$ constitute the characteristic vector associated with $p(A)$, normalized by the condition that $\sum_{i} x_{i}=1$. Hence

$$
p(A) x_{i}=\sum_{j=1}^{n} a_{i j} x_{j}, \quad i=1,2, \cdots, n .
$$

Thus

$$
p(A) \underset{i}{\operatorname{Min}} x_{i} \geqq\left(\operatorname{Min}_{i, j} a_{i j}\right) \sum_{j=1}^{n} x_{j}=\operatorname{Min}_{i, j} a_{i j} .
$$

On the other hand,

$$
p(A) \operatorname{Max}_{i} x_{i} \leqq \operatorname{Max}_{i} x_{i}\left(\operatorname{Max}_{i} \sum_{j=1}^{n} a_{i j}\right),
$$

whence 


$$
p(A) \leqq \operatorname{Max}_{i}\left(\sum_{j=1}^{n} a_{i j}\right) .
$$

Combining (5) and (7) we have

$$
\operatorname{Min}_{i} x_{i} \geqq \operatorname{Min}_{i, j} a_{i j} / \operatorname{Max}_{i}\left(\sum_{j=1}^{n} a_{i j}\right) .
$$

4. An alternative definition of $p(A)$. Let us now show, following the lead of Shapley, that we may define $p(A)$ as follows

LEMma 4. $p(A)$ is the unique solution of

$$
\lambda=\underset{R^{\prime}}{\operatorname{Max}} \operatorname{Min}_{i}\left[\sum_{j=1}^{n} a_{i j} x_{j}+\lambda\left(1-x_{i}\right)\right]
$$

or of

$$
\lambda=\operatorname{Min}_{R^{\prime}} \operatorname{Max}_{1}\left[\sum_{j=1}^{n} a_{i j} x_{j}+\lambda\left(1-x_{i}\right)\right]
$$

where $R^{\prime}$ is as defined by (3.2).

Proof. It is sufficient to prove that $p(A)$ satisfies (1). The proof of the other statement is similar. We have, for all $x$ in $R^{\prime}$,

$$
\lambda \geqq \operatorname{Min}_{i}\left[\sum_{j=1}^{n} a_{i j} x_{j}+\lambda\left(1-x_{i}\right)\right],
$$

for any solution $\lambda$, with equality for at least one $x$. We shall prove below that there is exactly one solution which may be obtained iteratively.

Hence, for all $x \in R^{\prime}$,

$$
0 \geqq \operatorname{Min}_{i}\left[\sum_{j=1}^{n} a_{i j} x_{j}-\lambda x_{i}\right] \text {, }
$$

or

$$
0 \geqq \operatorname{Min}_{i}\left[x_{i}\left\{\sum_{j=1}^{n} a_{i j} x_{j} / x_{i}-\lambda\right\}\right],
$$

for all $x \in R^{\prime}$. Since $x_{i}>0$, it follows that

$$
\lambda \geqq \operatorname{Min}_{i}\left(\sum_{j=1}^{n} a_{i j} x_{j} / x_{i}\right)
$$

for all $x$, with equality for one $x$, at least. Hence 


$$
\lambda=\operatorname{Max}_{R^{\prime}} \operatorname{Min}_{i}\left(\sum_{j=1}^{n} a_{i j} x_{j} / x_{i}\right)=p(A),
$$

which shows uniqueness provided we assume existence.

Similarly we may demonstrate the result in (2).

5. A nonlinear recurrence relation. Let us now consider the nonlinear recurrence relation

$$
u_{n+1}=\operatorname{Min}_{R^{\prime}} \operatorname{Max}_{i}\left[\sum_{j=1}^{n} a_{i j} x_{j}+u_{n}\left(1-x_{i}\right)\right],
$$

where $R^{\prime}$ is as above and $u_{0}$ is arbitrary. We shall prove

THEOREM.

$$
p(A)=\lim _{n \rightarrow \infty} u_{n}
$$

A similar result holds for the recurrence relation based upon (4.2). Proof. We have

$$
\begin{aligned}
u_{n+1} & =\underset{R^{\prime}}{\operatorname{Min}} \operatorname{Max}_{i}\left[\sum_{j=1}^{n} a_{i j} x_{j}+u_{n}\left(1-x_{i}\right)\right] \\
& =\underset{R^{\prime}}{\operatorname{Min}} \operatorname{Max}_{y}\left[\sum_{i=1}^{n} y_{i}\left[\sum_{j=1}^{n} a_{i j} x_{j}+u_{n}\left(1-x_{i}\right)\right]\right]
\end{aligned}
$$

where the maximum in $y$ is over the region $y_{i} \geqq 0, \sum_{i=1}^{n} y_{i}=1$. Using the min-max theorem of von Neumann, this may also be written

$$
u_{n+1}=\underset{y}{\operatorname{Max}} \operatorname{Min}_{R^{\prime}}\left\{\sum_{i=1}^{n} y_{i}\left[\sum_{j=1}^{n} a_{i j} x_{j}+u_{n}\left(1-x_{i}\right)\right]\right\} \text {. }
$$

Let us write this recurrence relation in the form

$$
u_{n+1}=\operatorname{Min}_{R^{\prime}} \operatorname{Max}_{y} T\left(u_{n}, x, y\right)=\underset{v}{\operatorname{Max}} \operatorname{Min}_{R^{\prime}} T\left(u_{n}, x, y\right) .
$$

Then using a device we have employed frequently in the theory of dynamic programming, cf. $[2 ; 3]$, we have

$$
\begin{aligned}
u_{n+1} & =T\left(u_{n}, \bar{x}, \bar{y}\right), \\
u_{n} & =T\left(u_{n-1}, x^{*}, y^{*}\right),
\end{aligned}
$$

where $(\bar{x}, \bar{y})$ and $\left(x^{*}, y^{*}\right)$ are respectively values where the min-max and max-min are assumed for $n$ and $n-1$ respectively.

Hence, by virtue of (5), 


$$
\begin{aligned}
u_{n+1}=T\left(u_{n}, \bar{x}, \bar{y}\right) & \geqq T\left(u_{n}, \bar{x}, y^{*}\right) \\
& \leqq T\left(u_{n}, x^{*}, \bar{y}\right),
\end{aligned}
$$

and

$$
\begin{aligned}
u_{n}=T\left(u_{n-1}, x^{*}, y^{*}\right) & \geqq T\left(u_{n-1}, x^{*}, \bar{y}\right) \\
& \leqq T\left(u_{n-1}, \bar{x}, y^{*}\right) .
\end{aligned}
$$

From this we obtain

$$
\begin{aligned}
u_{n+1}-u_{n} & \geqq T\left(u_{n}, \bar{x}, y^{*}\right)-T\left(u_{n-1}, \bar{x}, y^{*}\right) \\
& \leqq T\left(u_{n}, x^{*}, \bar{y}\right)-T\left(u_{n-1}, x^{*}, \bar{y}\right),
\end{aligned}
$$

which yields

$$
\begin{aligned}
u_{n+1}-u_{n} & \geqq\left(u_{n}-u_{n-1}\right) \sum_{i=1}^{n} y_{i}^{*}\left(1-\bar{x}_{i}\right) \\
& \leqq\left(u_{n}-u_{n-1}\right) \sum_{i=1}^{n} \bar{y}_{i}\left(1-x_{i}^{*}\right) .
\end{aligned}
$$

Hence

$$
\begin{aligned}
& \left|u_{n+1}-u_{n}\right| \\
& \quad \leqq\left|u_{n}-u_{n-1}\right| \operatorname{Max}\left[\sum_{i=1}^{n} y_{i}^{*}\left(1-\bar{x}_{i}\right), \sum_{i=1}^{n} \bar{y}_{i}\left(1-x_{i}^{*}\right)\right] .
\end{aligned}
$$

Since $\bar{x}_{i}, x_{i}^{*} \geqq d>0$, and $y_{i} \geqq 0, \sum_{i} y_{i}=1$, we have

$$
\left|u_{n+1}-u_{n}\right| \leqq(1-d)\left|u_{n}-u_{n-1}\right|,
$$

and hence geometric convergence of $\sum_{w=0}^{\infty}\left(u_{n+1}-u_{n}\right)$. The limit of $u_{n}$ exists, and must equal $p(A)$. Observe that this is a situation where only the value of a game is of interest, if we wish only to determine $p(A)$. Consequently, the iterative procedure of [4] may be of some merit here.

6. Monotone convergence. If we set

$$
u_{0}=\operatorname{Min}_{R^{\prime}} \operatorname{Max}_{i} \sum_{j} a_{i j} x_{j},
$$

we see that $u_{1} \geqq u_{0}$ and hence $u_{n+1} \geqq u_{n}$, which ensures monotone convergence.

Similarly, if we have

$$
a_{1} \leqq a_{i j} \leqq a_{2},
$$

and use the Perron roots of the associated matrices as initial ap- 
proximations, we obtain monotone increasing and monotone decreasing sequences respectively.

\section{BIBLIOGRAPHY}

1. R. Bellman, An introduction to the theory of dynamic programming, RAND Report No. R-245, 1953.

2. - A survey of the theory of time-lag, retarded control and hereditary processes, RAND Report No. R-271, 1954.

3. - Some functional equations in the theory of dynamic programming-I, Trans. Amer. Math. Soc. vol. 80 (1955) pp. 51-71.

4. - On a new iterative algorithm for finding the solutions of games and linear programming problems, Symposium on Monte Carlo Methods, University of Florida, March, 1954.

5. R. Bellman and J. LaSalle, Non-zero sum games and stochastic processes, RAND Memorandum No. RM-271, 1949.

6. L. Shapley, Stochastic games, Proc. Nat. Acad. Sci. U.S.A. vol. 39 (1953) pp. 1095-1100.

7. J. von Neumann, Ergebnisse eines Math. Kolloquiums vol. 8 (1937) pp. 73-78.

8. J. von Neumann and O. Morgenstern, Theory of games and economic behavior, Princeton University Press, 1944.

The Rand Corporation 